\title{
Robust Unmixing of Dynamic Sequences Using Regions of Interest (RUDUR)
}

\author{
Marc Filippi*, Michel Desvignes, and Eric Moisan
}

\begin{abstract}
In dynamic planar imaging, extraction of signals specific to structures is complicated by structures superposition. Due to overlapping, signals extraction with classic regions of interest (ROIs) methods suffers from inaccuracy, as extracted signals are a mixture of targeted signals. Partial volume effect raises the same issue in dynamic tomography. Source separation methods such as factor analysis of dynamic sequences, have been developped to unmix such data. However the underlying problem is underdetermined and the model used is not relevant in the whole image. This non-uniqueness issue was overcome by introducing prior knowledge, such as sparsity or smoothness, in the separation model. In pratice, these methods are barely used because of the lack of reliability of their results. Previously developed methods aimed to be fully automatic, but efficiency can be improved with additional prior knowledge. Some methods using ROIs knowledge in a straightforward way have been proposed. In this paper, we propose an unmixing method, based on an objective function minimization and integrating these ROIs in a different and robust manner. The objective function promotes consistent solutions regarding ROIs while relaxing the model outside ROIs. In order to reduce user-dependent effects, ROIs are used as soft constraints in a robust way through the use of a distance matrix. Consistency, effectiveness and robustness to the ROIs selection are demonstrated on a toy example, a highly realistic simulated renography dataset and a clinical dataset. Performance is compared with competitive methods.
\end{abstract}

Index Terms-Nuclear imaging, blind source separation, inverse methods, kidney, computer-aided detection and diagnosis.

\section{INTRODUCTION}

$\mathbf{N}$ UCLEAR imaging is a physiological imaging modality dedicated to the assessment of organ and tissue function. The first step of the pipeline is to acquire the data, a sequence of $2 \mathrm{D}$ or $3 \mathrm{D}$ images, representing the dynamic activity of a tracer injected inside the patient's body. The second step is to extract the time activity curve (TAC) of each organ and tissue of interest. These curves are the input of a compartmental model in the third step, giving kinetic parameters as outputs. Finally, each organ and tissue function is assessed by a simple analysis of these kinetic parameters. This paper is focused on the second step, the extraction of TACs. The most straightforward method for the extraction consists in defining a region of interest (ROI) for each structure (organ or tissue), and then to average the activity inside each ROI. For each structure, a TAC is obtained. However, this method is user-dependent and often produces incorrect TACs, especially in scintigraphy, where the

$*$ M. Filippi, is with Univ. Grenoble Alpes, CNRS, Grenoble INP ${ }^{\dagger}$, GIPSAlab, 38000 Grenoble, France, e-mail: marc.filippi@gipsa-lab.grenoble-inp.fr

M. Desvignes and E. Moisan, are with Univ. Grenoble Alpes, CNRS, Grenoble INP ${ }^{\dagger}$, GIPSA-lab, 38000 Grenoble, France

$\dagger$ Institute of Engineering Univ. Grenoble Alpes
2D-images are projections of 3D-structures. As projections of structures often overlap, a ROI is likely to contain the activity of several structures, resulting in an incorrect TAC estimation. An alternative method is to draw a very small ROI containing only one structure, but this often produces noisy TACs and the method is not always applicable. It is therefore necessary to perform spatial and temporal structure unmixing in order to obtain the pure TACs [1]. Because of partial volume effect, this problem is also adressed in other medical imaging modalities, such as SPECT [2], [3], PET [4], [5], contrast-enhanced MRI [6], or constrast enhanced ultrasound [7], [8].

Factor analysis based methods have been developed to perform this unmixing [1], [9]. These methods share the same linear mixing model as positive matrix factorization [10] or more popular non-negative matrix factorizaton [11]. Data are assumed to be a mixture of a small number of structures, each structure having its own positive spatial and temporal signature. The objective is to recover the spatial and temporal signature of each structure. However, a major drawback of the factor analysis model is that the solution is not mathematically unique and may have no physiological meaning [12], [13]. In order to obtain physiologically meaningful solutions and to overcome non-uniqueness issues, prior knowledges on data have been introduced.

Parametric methods have been developed, with various assumptions on the TACs shape [14], [15]. The TACs are decomposed on a dictionary of predefined parametrized temporal curves. The degrees of freedom are then reduced since only few parameters have to be computed. The major drawback of these methods is the lack of flexibility, and the choice of the dictionary.

A regularization constraint on the source images representing structures position has been introduced in [16]. Unfortunately, the separation is not completely achieved as some structures remain mixed. To overcome this problem and nonuniqueness issues, a penalized least squares function was proposed in [17]. The function introduces a new term penalizing solutions for which pixels contain a mixture of structures. The main drawback of this method is that it penalizes overlaps, which is the essence of data, and therefore penalizes the target solution.

More recently, some methods using physiological model assumptions have been developed, where TACs are assumed to be a convolution of an input function and tissue kernels. These methods extract both TACs and convolution kernels. In [6] the input function and convolution kernels are parametrized as exponential functions, but the method shares the same flexibility issue as parametrized methods described above. This 
issue was corrected in [18] and [19] by assuming only sparsity on the convolution kernels. However, these methods based on a variational Bayesian algorithm can be rather complex and time consuming. Moreover, the separation is not always achieved due to the presence of local minima.

A few methods using ROIs knowledge have also been proposed. In [20], ROIs are used as masks to constrain the source images to be null outside their ROIs. Thanks to these constraints, source images are first estimated and then used to compute TACs. However, the non-negativity criterion on TACs is no longer ensured in this method and may lead to inconsistent results with negative values on the TACs. It was shown in [13] that the non-uniqueness issue can be overcome using ROIs as masks. A generic method using spatial and temporal prior knowledge was proposed in [21]. With this method, it is possible to ensure non negativity of source images and TACs. Also ROIs cans be used as masks to constrain the source images. However, in this method ROIs can only be used as hard constraints for source images. In [22], a method using probabilistic ROI is also used, but with an automatic ROIs selection. This method does not allow ROIs to be considered as prior knowledge.

In all previous methods, the underlying separation model is assumed to be true in the whole image or in a selected rectangular window. This assumption is however wrong on clinical data and must be relaxed. Unmixing remains a challenging problem in nuclear medicine, especially in scintigraphy. The nuclear medicine context is nonetheless very specific because prior knowledge is easily accessible.

In order to improve unmixing, our proposed method robustly integrate ROIs knowledge as soft constraints, by generalizing and improving a previous work described in [23]. This method introduced a criterion based on the distance to ROI. In this paper, we propose to generalize this criterion and to robustly relax data fidelity constraint outside ROIs. The objective function optimization is improved in term of speed. Details and rational behind the choice of the objective function will be given in section II. Implementation details are given in section III. The proposed method is compared to other competitive methods in section IV. Consistency, effectiveness and robustness to ROIs selection are demonstrated on a toy example, on a highly realistic simulated renography dataset and also on a clinical data. These datasets have already been used in [19].

\section{MOdel AND METHOD}

The linear mixing model used to describe the data is first presented. The new objective function is introduced, along with the reasons for choosing each criterion.

\section{A. Linear Mixing Model}

In nuclear imaging, the data represents the dynamic activity of a tracer as an image sequence. Each image is then an approximation of the tracer concentration in the patient's body during a time frame. In nuclear imaging, image sequences are commonly modeled by the linear mixing model. This model assumes that the dynamic activity of each pixel is described by

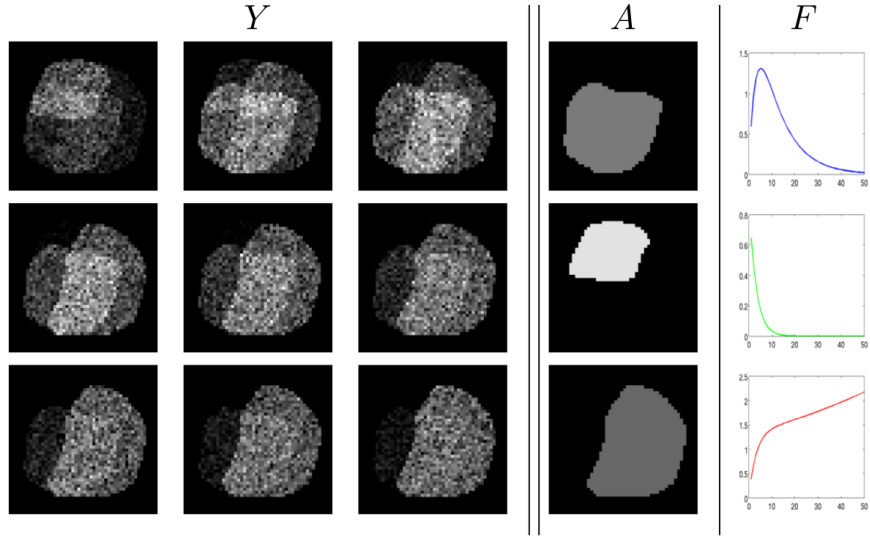

Fig. 1. An example of matrix $Y, A$ and $F$ consistent with the model described in equations (1) and (2), with $K=3$. The first three columns contain the data (matrix $Y$ ) at time $\mathrm{t}=0,3,6 \ldots 24$. The fourth column contains the source images $(A)$, and the last column contains the corresponding factors $(F)$. The $\mathrm{x}$-axis represents the tim and the $\mathrm{y}$-axis the activity.

a linear combination of only few TACs (also called factors), which are shared by all pixels. These factors represent the dynamic activity of the physiological structures present in the image. Formally, we get

$$
Y_{i, t}=\sum_{k=1}^{K} A_{i, k} F_{k, t}+\epsilon_{i, t} .
$$

where $Y$ is the $N$ by $P$ matrix containing the image sequence, with $N$ the number of pixels, $P$ the number of frames, and $K$ the number of observed factors. Each column of $Y$ corresponds to the vectorized image of the tracer activity during a time frame. $Y_{i, t}$ is thus the activity on the $i^{t h}$ pixel in the $t^{t h}$ frame. The matrix $F$ of size $K$ by $P$ is the matrix of factors. Its $k^{t h}$ row corresponding to the $k^{\text {th }}$ factor activity. The matrix $A$ of size $N$ by $K$ contains the weights of the linear combinations. The $k^{t h}$ column of $A$ thus corresponds to a vectorized image representing the weight of the $k^{t h}$ factor. Finally, $\epsilon$ is a matrix of the same size as $Y$ representing the noise. Using a more compact matrix notation we obtain

$$
Y=A \times F+\epsilon
$$

An example of matrices $Y, A$ and $F$ consistent with this model is given in Figure 1.

In this work, the goal is to recover source images (matrix $A$ ) and factors (matrix $F$ ) from data (matrix $Y$ ). In order to obtain a physiologically meaningful solution, positivity constraints are added to the coefficients of $A$ and $F$. Since $F$ represents structures activity and $A$ represents structures weight along the image, their coefficients must be positive, i.e.

$$
\begin{aligned}
& \forall i=1 . . N, k=1 . . K, A_{i, k} \geq 0 \\
& \forall k=1 . . K, t=1 . . P, F_{k, t} \geq 0
\end{aligned}
$$

A common way to determine $A$ and $F$ under these positivity constraints is to compute the least squares solution, as realized in classical non negative matrix factorization method [11]. However, this problem is underdetermined since the solution is not unique [12], [13]. To overcome this issue, we introduce 
on the next part a new criteria based on ROIs knowledge. It should be mentionned that the previous model gives a good approximation for only a limited area of the image. Our method robustly uses ROIs knowledge to relax the linear mixing model constraint outside the selected ROIs.

\section{B. Objective Function}

The proposed objective function contains three terms. The first is a relaxed data fidelity term and the others introduce spatial prior knowledge (on $A$ ) and temporal prior knowledge (on $F$ ). The first two terms use ROIs knowledge. A ROI is a set of pixels assumed to contain a structure. In this paper, ROIs are represented by a $N$ by $K$ matrix $M$ (i.e. same size as $A$ ) containing binary masks. A column of $M$ is a vectorized binary image representing the ROI of one structure, with

$$
M_{i, k}= \begin{cases}1 & \text { if the } i^{t h} \text { pixel is in the } k^{t h} \mathrm{ROI} \\ 0 & \text { otherwise }\end{cases}
$$

However, the key knowledge of ROI is not its binary information (i.e. whether or not a pixel belongs to this ROI), as it is used in the basic averaging method. Pixels close to a ROI are treated in the same way than distant pixels. This makes the method highly sensitive to user choices. In order to make the method more robust to ROI selection, the ROI knowledge should not be only used associated with the binary matrix $M$. To overcome this limitation, we introduce the distance to ROIs matrix $D$ of the same size as $M(N$ by $K)$, defined by

$$
D_{i, k}=\min _{j / M_{j, k}=1} \operatorname{dist}_{e u c}(i, j)
$$

where $\operatorname{dist}_{\text {euc }}(i, j)$ is the euclidean distance between the $i^{\text {th }}$ and the $j^{t h}$ pixel. This matrix contains the euclidean distance from each pixel to each ROI. Specifically, $D_{i, k}=0$ if and only if the $i^{t h}$ pixel is in the $k^{t h}$ ROI. This matrix will be used to express constraints and relaxation.

The proposed objective function, to be minimized, is expressed

$$
f_{\mathrm{RUDUR}}(A, F)=f_{\mathrm{WLS}}(A, F)+\alpha f_{\mathrm{ROI}}(A)+\beta f_{\mathrm{Tik}}(F)
$$

with $\alpha$ and $\beta$ two non-negative constants. The objective function is decomposed in three terms. The first term, $f_{\mathrm{WLS}}$, is a weighted least squares term ensuring data fidelity, and containing a model relaxation thanks to matrix $D$. The second term, $f_{\text {ROI }}$, penalizes solutions with inconsistent coefficients in $A$ according to ROIs using matrix $D$. Finally, the third term $f_{\text {Tik }}$ is a Tikhonov regularization term promoting solutions with smooth TACs in $F$.

More precisely, the data fidelity criterion $f_{\mathrm{WLS}}$ is expressed as

$$
f_{\mathrm{WLS}}(A, F)=\|W(Y-A \times F)\|_{F}^{2}
$$

where $\|.\|_{F}$ is the Frobenius norm, and $W$ is a $N$ by $N$ diagonal weighting matrix relaxing data fidelity, with

$$
W_{i, i}=\frac{1}{1+\min _{k=1}^{K} g\left(D_{i, k}\right)}
$$

where function $g$ can be chosen as

$$
g(x)=\gamma \times x^{2}
$$

with $\gamma$ a non negative constant. While data fidelity is maintained for pixels inside one of the ROIs, data fidelity of pixels outside of ROIs is relaxed according to the distance to the closest ROI. The farther the ROI, the greater the relaxation will be. Indeed, a pixel far from all ROIs is likely to not contain any information about TACs of interest. In order to avoid these far pixels to have a large impact on the separation process, the constraint on their data fidelity must be relaxed. Robustness to ROIs selection is thus obtained by relaxing data fidelity according to the distance to the closest ROI. When $\gamma=0$, $f_{\mathrm{WLS}}$ is equal to the classic least squares fidelity criterion without relaxation.

The second term also uses ROIs knowledge through the matrix $D$. A pixel far from one ROI should not contain the corresponding factor. The corresponding coefficient in the matrix $A$ is thus expected to be close to $0 . f_{\mathrm{ROI}}$ penalizes the opposite behaviour with

$$
f_{\mathrm{ROI}}(A)=\|h(D) \circ A\|_{1, \mu}
$$

where $\circ$ is the Hadamard product and $\mu$ a small positive constant. For the penalization to increase with the distance, the function $h$ is chosen as

$$
h(D)=D \circ D
$$

$\|\cdot\|_{1, \mu}$ is a smooth $\ell 1$-norm defined as

$$
\|X\|_{1, \mu}=\sum_{i=1}^{N} \sum_{k=1}^{K} \sqrt{X_{i, k}^{2}+\mu^{2}}-\mu
$$

where $X$ is a $N$ by $P$ matrix. Unlike classic $\ell 1$-norm, this smooth $\ell 1$-norm is differentiable at 0 which is more convenient for the optimization process. Pixels far from a ROI are not expected to contain the corresponding factor. The farther the pixel, the lower the likelihood. This knowledge is included here through the use of the distance to ROIs matrix $D . f_{\text {ROI }}$ promotes consistent solutions according to ROIs. Once again, robustness to the ROIs selection is ensured through the use of matrix $D$

If the weighting constant $\alpha$ is chosen to be $+\infty$, the coefficients of matrix $A$ are constrained to be equal to 0 when the pixel is not in the corresponding ROI (i.e $M_{i, k}=0 \Rightarrow$ $A_{i, k}=0$ ). This particular case was exploited in a previous work [24].

Finally, the third term $f_{\text {Tik }}$ is a Tikhonov regularization. Targeted TACs are expected to be smooth. However, in a noisy context, separation methods often produces noisy TACs. In order to avoid this behaviour, this term promotes solutions containing smooth TACs in $F$. The function is expressed

$$
f_{\text {Tik }}(F)=\left\|\Gamma \times\left(F^{T}\right)\right\|_{F}^{2}
$$


where ${ }^{T}$ is the transpose operator and $\Gamma$ is a lower bi-diagonal $P$ by $P$ gradient operator matrix

$$
\Gamma=\left[\begin{array}{ccccc}
1 & 0 & \ddots & \ddots & 0 \\
-1 & 1 & 0 & \ddots & \ddots \\
0 & \ddots & 1 & 0 & \ddots \\
\ddots & 0 & -1 & \ddots & 0 \\
0 & \ddots & 0 & -1 & 1
\end{array}\right]
$$

The minimization of $f_{\text {RUDUR }}$ according to $A$ and $F$ and under positivity constraints (3) and (4) gives the desired TAC and source image of each structure of interest in $\tilde{F}$ and $\tilde{A}$ respectively. It is expressed by

$$
(\tilde{A}, \tilde{F})=\underset{A \geq 0, F \geq 0}{\arg \min } f_{\text {RUDUR }}(A, F)
$$

The minimization of this objective function $f_{\text {RUDUR }}$ is detailed in the next section.

\section{IMPLEMENTATION}

Due to the least squares term in $f_{\mathrm{WLS}}$, the objective function $f_{\text {RUDUR }}$ is not jointly convex in $A$ and $F$. However, $f_{\text {RUDUR }}$ is convex in $A$ and convex in $F$. Under this condition, the classic way to minimize $f_{\text {RUDUR }}$ is to use an alternate minimization method. The basic procedure for this type of method is to alternate minimization according to $A$ (with $F$ fixed) and minimization according to $F$ (with $A$ fixed) until convergence. Several iterations are performed for each minimization before switching the roles of $A$ and $F$. The proposed method relies on the alternating non negative least squares framework (ANLS, [25]). Conjugate gradient descent with the Polak-Ribière formula [26] was used to find the descent direction at each iteration. The step can be chosen to be constant over the iterations, or proportional to the inverse of the current Lipschitz constant of the partial derivative functions. In this case, the Lipschitz constant is different for each pixel and each iteration (see Appendix B). In practice, this accelerates the convergence of the algorithm.

The positivity of the coefficients of $A$ and $F$ is ensured by a projection on the positive orthant at each iteration of the optimization. To increase the speed of the algorithm, $A$ is initialized as the matrix of binary masks $M$, and $F$ as the average activity inside each mask.

To overcome identification problems, $A$ is normalized after each iteration so that the sum of each source image is equal to the ratio $\frac{N}{K}$. For the matrix product $F_{k} \times A_{k}$ to remain unchanged, $F$ is counter normalized and

$$
\begin{gathered}
\forall k: F_{k}=F_{k} \times \sum_{n=1}^{N} A_{n, k} \times \frac{K}{N} \\
\forall k: A_{k}=\frac{A_{k}}{\sum_{n=1}^{N} A_{n, k}} \times \frac{N}{K}
\end{gathered}
$$

The complete optimization pipeline is detailed in Algorithm
Details about partial derivatives of the objective function are presented in Appendix A. The Condition in the while loop is set to false if and only if the relative change in the objective function is lower than $10^{-7}$ three times in a row. The presented results have been obtained with the constant nbIter set to 5 . The functions $g$ and $h$ are chosen as suggested in equations (10) and (12), with $\gamma=3$ and $\mu=0.1$. The number of factors $K$ is assumed to be known. The matrix $W$ is a diagonal matrix of size $N$, where $N$ is the number of pixels. Due to the generally large size of $N$, it is computationally more efficiency to treat diagonal elements of $W$ separately instead of performing a matrix product. In the next section, unmixing is achieved by the proposed method and each simulation was performed in only a few seconds (using a $2.60 \mathrm{GHz} \mathrm{CPU}$ ).

The results of the next section are obtained using the RUDUR method with $\alpha=1$ and $\beta=10$. Better results can be obtained with other parameters values, but usually optimal parameters are not available without ground truth. However, as RUDUR is not too sensitive to the choice of parameters, it is possible to build a model of the dataset of interest and find rough values for parameters thanks to the ground truth of the model.

A MATLAB implementation of the algorithm and some demos are available for download at http://www.gipsalab.grenoble-inp.fr/ marc.filippi/.

\section{RESULTS}

Unmixing results of the proposed algorithm $(R U D U R)$ are compared with state of the art methods, on simulated and clinical dataset which have already been used in [19]. The competing methods considered in this evaluation are :

1) $F_{P L S}$ [17]: In this method, unmixing is achieved thanks to the minimization of an objective function, containing a classic data fidelity criterion, a term penalizing solutions with negative coefficients, and a term penalizing overlapping in source images. In the following results, this objective function is optimized with Algorithm 1 and the term penalizing solutions with negative coefficients is ignored.

2) FAMIS [21]: This method concerns Benali's approach, a generic method that can use temporal and spatial prior knowledge. For the simulations, the method considered the same ROIs as the ones selected in RUDUR. Source images were forced to have null coefficients outside their corresponding ROI.

3) FAROI [22]: This method promotes sparsity on source images, and integrates automatic estimation of probabilistic ROI which facilitates model solving. The model is solved with the variational Bayesian method [27].

4) S-BSS-vecDC [19]: This method uses physiological model assumptions. TACs are assumed to be a convolution of the input function and a tissue kernel. The method promotes sparse source images and sparse convolution kernels, and also uses the variational Bayesian method.

For FAROI and S-BSS-vecDC, results were obtained using the MATLAB code available at http://www.utia.cas.cz/AS/softwaretools/image_sequences 1 . 

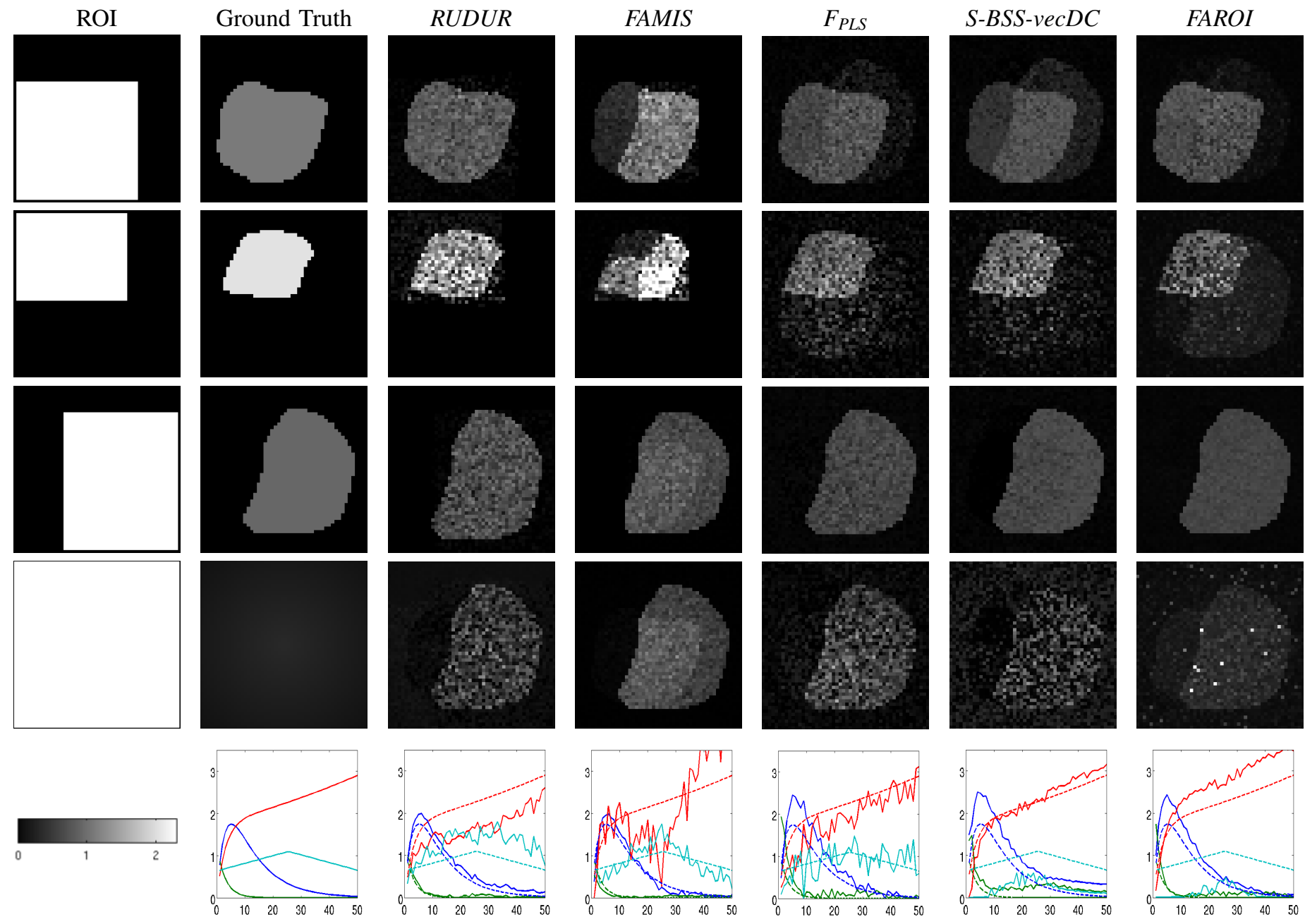

Fig. 2. Unmixing results on one simulation of the toy example, with $\sigma=0.5$. The first four rows contain ground truth (second column) and an estimation of the source images for each algorithm (columns 3 to 7). ROIs used for RUDUR and FAMIS are shown on the first column. The colorbar used for source images display is on the bottom left of the figure. The last row contains ground truth (second column) and the estimation of TACs $(F)$ for each algorithm (columns 3 to 7). For columns 3 to 7, estimations are depicted in solid lines, and ground truth in dotted lines, with time on the x-axis and activity on the y-axis.

TABLE I

ESTIMATION ERRORS ON FACTORS $(F)$ - TOY EXAMPLE

\begin{tabular}{|c|c|c|c|c|c|c|c|c|c|}
\hline & & \multicolumn{4}{|c|}{ sigma $=0.5$} & \multicolumn{4}{|c|}{ sigma $=1$} \\
\hline Measure & Algorithm & F1 & F2 & F3 & F4 & F1 & $\mathrm{F} 2$ & F3 & $\mathrm{F} 4$ \\
\hline \multirow{5}{*}{ NMSE } & 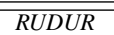 & $\overline{0.039 \pm 0.006}$ & $\overline{00.079 \pm 0.018}$ & $\overline{0.075 \pm 0.004}$ & $\overline{0.270 \pm 0.018}$ & $0.035 \pm 0.008$ & $0.367 \pm 0.075$ & $0.087 \pm 0.015$ & $0.460 \pm 0.082$ \\
\hline & FAMIS & $0.033 \pm 0.013$ & $0.143 \pm 0.031$ & $0.117 \pm 0.036$ & $0.082 \pm 0.010$ & $0.220 \pm 0.295$ & $0.476 \pm 0.278$ & $0.421 \pm 0.195$ & $0.645 \pm 0.158$ \\
\hline & $F_{P L S}$ & $0.167 \pm 0.027$ & $2.217 \pm 0.296$ & $0.036 \pm 0.011$ & $0.202 \pm 0.063$ & $0.540 \pm 0.069$ & $26.72 \pm 4.569$ & $0.185 \pm 0.050$ & $0.711 \pm 0.123$ \\
\hline & $S-B S S$ & $0.402 \pm 0.119$ & $2.308 \pm 0.600$ & $0.015 \pm 0.012$ & $0.708 \pm 0.154$ & $0.540 \pm 0.249$ & $8.650 \pm 3.641$ & $0.125 \pm 0.054$ & $0.360 \pm 0.125$ \\
\hline & FAROI & $0.129 \pm 0.019$ & $0.932 \pm 0.077$ & $0.051 \pm 0.003$ & $0.843 \pm 0.016$ & $0.030 \pm 0.056$ & $2.136 \pm 0.343$ & $0.091 \pm 0.115$ & $0.637 \pm 0.041$ \\
\hline \multirow{5}{*}{ NMAE } & RUDUR & $0.264 \pm 0.023$ & $0.610 \pm 0.091$ & $0.268 \pm 0.007$ & $0.474 \pm 0.017$ & $0.187 \pm 0.024$ & $1.281 \pm 0.168$ & $0.244 \pm 0.024$ & $\overline{0.530 \pm 0.052}$ \\
\hline & FAMIS & $0.191 \pm 0.042$ & $0.727 \pm 0.065$ & $0.281 \pm 0.043$ & $0.236 \pm 0.016$ & $0.404 \pm 0.239$ & $1.181 \pm 0.233$ & $0.541 \pm 0.135$ & $0.722 \pm 0.111$ \\
\hline & $F_{P L S}$ & $0.416 \pm 0.039$ & $2.647 \pm 0.277$ & $0.150 \pm 0.022$ & $0.370 \pm 0.066$ & $0.664 \pm 0.054$ & $8.952 \pm 1.072$ & $0.358 \pm 0.065$ & $0.693 \pm 0.055$ \\
\hline & $S-B S S$ & $0.658 \pm 0.037$ & $3.694 \pm 0.465$ & $0.095 \pm 0.026$ & $0.709 \pm 0.021$ & $0.775 \pm 0.209$ & $6.418 \pm 1.635$ & $0.317 \pm 0.080$ & $0.529 \pm 0.089$ \\
\hline & FAROI & $0.375 \pm 0.036$ & $1.679 \pm 0.068$ & $0.216 \pm 0.008$ & $0.824 \pm 0.006$ & $0.148 \pm 0.085$ & $2.676 \pm 0.260$ & $0.261 \pm 0.087$ & $0.758 \pm 0.018$ \\
\hline
\end{tabular}

Results are in the form mean \pm std where mean and $s t d$ are the average and the standard deviation of the current measure (NMSE or NMAE) over a set of 100 simulation

of the toy example. S-BSS corresponds to $S$-BSS-vecDC method. 
TABLE II

ESTIMATION ERRORS ON SOURCE IMAGES $(A)$ - TOY EXAMPLE

\begin{tabular}{|c|c|c|c|c|c|c|c|c|c|}
\hline \multirow[b]{2}{*}{ Measure } & \multirow[b]{2}{*}{ Algorithm } & \multicolumn{4}{|c|}{ sigma $=0.5$} & \multicolumn{4}{|c|}{ sigma $=1$} \\
\hline & & A1 & A2 & A3 & A4 & A1 & A2 & A3 & A4 \\
\hline \multirow{5}{*}{ NMSE } & $R U D U R$ & $0.047 \pm 0.003$ & $0.168 \pm 0.013$ & $0.086 \pm 0.010$ & $0.805 \pm 0.044$ & $0.156 \pm 0.014$ & $0.446 \pm 0.029$ & $0.236 \pm 0.022$ & $1.982 \pm 0.094$ \\
\hline & FAMIS & $0.252 \pm 0.006$ & $0.597 \pm 0.027$ & $0.061 \pm 0.003$ & $1.396 \pm 0.014$ & $0.811 \pm 1.117$ & $1.133 \pm 0.365$ & $0.222 \pm 0.429$ & $3.132 \pm 2.408$ \\
\hline & $F_{P L S}$ & $0.103 \pm 0.048$ & $0.344 \pm 0.013$ & $0.044 \pm 0.007$ & $1.190 \pm 0.089$ & $0.285 \pm 0.046$ & $0.727 \pm 0.112$ & $0.090 \pm 0.030$ & $1.157 \pm 0.067$ \\
\hline & $S-B S S$ & $0.603 \pm 0.401$ & $0.632 \pm 0.322$ & $0.025 \pm 0.003$ & $1.242 \pm 0.200$ & $0.519 \pm 0.228$ & $0.922 \pm 0.197$ & $0.116 \pm 0.065$ & $1.232 \pm 0.184$ \\
\hline & FAROI & $0.079 \pm 0.002$ & $0.389 \pm 0.009$ & $0.032 \pm 0.001$ & $1.094 \pm 0.008$ & $0.272 \pm 0.016$ & $0.593 \pm 0.055$ & $0.034 \pm 0.010$ & $0.854 \pm 0.378$ \\
\hline \multirow{5}{*}{ NMAE } & RUDUR & $0.201 \pm 0.006$ & $0.404 \pm 0.016$ & $0.261 \pm 0.015$ & $0.683 \pm 0.015$ & $0.374 \pm 0.016$ & $0.667 \pm 0.023$ & $0.577 \pm 0.027$ & $1.143 \pm 0.014$ \\
\hline & FAMIS & $0.456 \pm 0.006$ & $0.676 \pm 0.013$ & $0.188 \pm 0.005$ & $1.081 \pm 0.007$ & $0.676 \pm 0.297$ & $0.891 \pm 0.091$ & $0.329 \pm 0.242$ & $1.267 \pm 0.107$ \\
\hline & $F_{P L S}$ & $0.393 \pm 0.057$ & $0.891 \pm 0.021$ & $0.269 \pm 0.012$ & $0.851 \pm 0.027$ & $0.644 \pm 0.050$ & $1.342 \pm 0.107$ & $0.347 \pm 0.044$ & $0.879 \pm 0.027$ \\
\hline & $S-B S S$ & $0.869 \pm 0.413$ & $1.216 \pm 0.371$ & $0.197 \pm 0.012$ & $0.933 \pm 0.082$ & $0.870 \pm 0.233$ & $1.517 \pm 0.201$ & $0.355 \pm 0.094$ & $0.853 \pm 0.052$ \\
\hline & FAROI & $0.375 \pm 0.004$ & $1.023 \pm 0.012$ & $0.264 \pm 0.002$ & $0.933 \pm 0.004$ & $0.691 \pm 0.032$ & $1.286 \pm 0.079$ & $0.237 \pm 0.034$ & $0.619 \pm 0.180$ \\
\hline
\end{tabular}
of the toy example. $S$ - $B S S$ corresponds to $S$ - BSS-vecDC method.

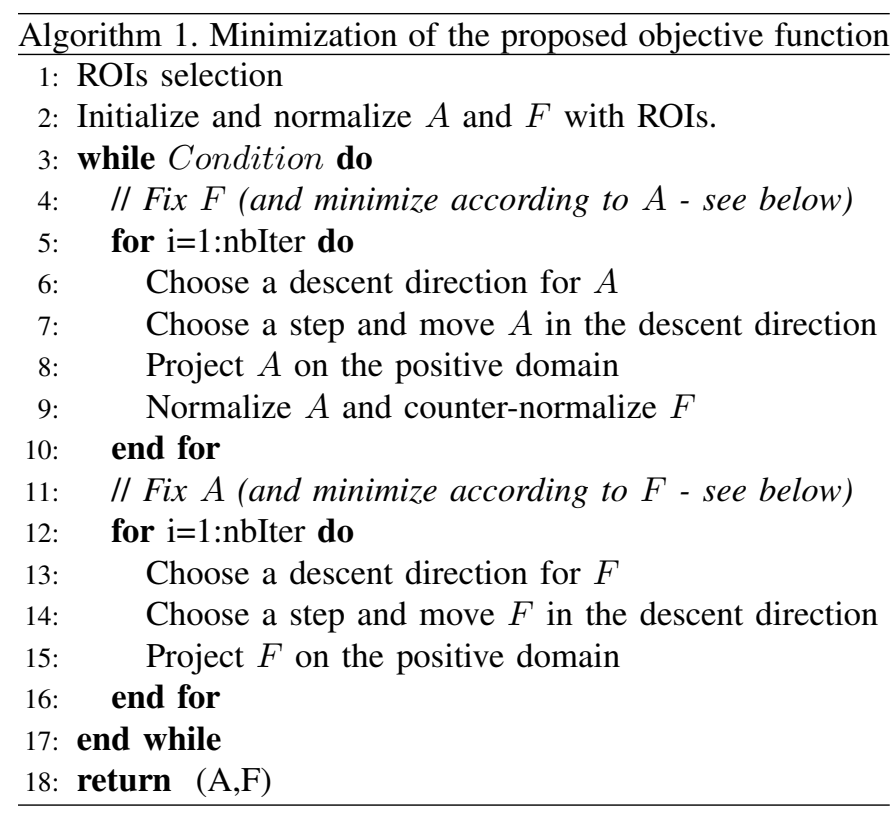

Methods are quantitatively compared with the normalized mean square error (NMSE) and the normalized mean average error (NMAE) on each TAC (rows of $F$ ) and on each source image (columns of $A$ ). In order to avoid scaling indeterminacy, each source image (column of $A$ ) is previously normalized so that its sum is equal to $\frac{N}{K}$. The corresponding TAC (row of $F$ ) is counter-normalized accordingly.

$$
\begin{gathered}
N M S E\left(F_{k}^{G T}, \hat{F}_{k}\right)=\frac{\sum_{t=1}^{P}\left(F_{k, t}^{G T}-\hat{F}_{k, t}\right)^{2}}{\sum_{t=1}^{P}\left(F_{k, t}^{G T}\right)^{2}} \\
N M A E\left(F_{k}^{G T}, \hat{F}_{k}\right)=\frac{\sum_{t=1}^{P}\left|F_{k, t}^{G T}-\hat{F}_{k, t}\right|}{\sum_{t=1}^{P}\left|F_{k, t}^{G T}\right|}
\end{gathered}
$$

where $F_{k}^{G T}$ is the ground truth TAC of the $k^{t h}$ factor, and $\hat{F}_{k}$ is the estimated one. A similar formula stands for source images comparison.

In practice, it is not possible to find the optimal parameters and ROIs for RUDUR and FAMIS. Consequently both of these methods are evaluated under suboptimal conditions in the following examples.

\section{A. Toy example}

Image sequences of this first dataset contain 50 images of size $50 \times 50$ pixels, and four factors $(K=4)$. The first three curves represent the convolution between a common input function and a kernel, thereby satisfying the $S$-BSS$v e c D C$ method assumptions. In order to be more realistic, a background factor has been considered. Source images and TACs are shown in Figure 2, column 2. More details about this dataset can be found in [19]. While the noise addition was done in the same way as [19], two different noise levels were considered for the simulations on the dataset. Also, the result presented is a summary of 100 realizations of the noise (instead of only one). Variance of noise is proportional to the signal, with $Y_{i, t}=Y_{i, t}+\sigma \times e_{i, t} \sqrt{Y_{i, t}}$, where $e_{i, t}$ is a random realization of a normal distribution with zero mean and standard deviation equal to $1 . \sigma$ is chosen equal to 0.5 or 1 and defines the noise level.

Results are detailed in Tables I and II. An example of results obtained for each method and with a noise level $\sigma=0.5$ is shown in Figure 2. For RUDUR and FAMIS, ROIs were manually chosen as rough rectangular windows, as depicted in the first column of Figure 2. The ROI of the background factor (the fourth factor) is selected as the union of the three other ROIs. For all the methods, the number of factors $K$ is set to 4 , and one hundred simulations are performed. Performance of the $F_{P L S}$ algorithm was improved by considering an initialization with ROI knowledge. This algorithm is really sensitive to initialization, and unmixing may fail when not considering ROI-based initialization.

Thanks to ROIs knowledge, RUDUR generally outperforms others methods in term of NMSE and NMAE for both TACs and source images. The source images estimation computed with other methods are corrupted by noise outside of their proper location, especially for sources 1 and 2. Since TACs and source images are jointly estimated, this noise also affects TACs estimation. Thanks to ROIs knowledge, extraction of source images are improved with RUDUR by limiting the possibility of unreal estimations. TACs estimations also benefit from this improvement. The low standard deviation obtained indicates that the proposed method leads to consistent unmixing for all simulations. FAMIS is the only other method leading 

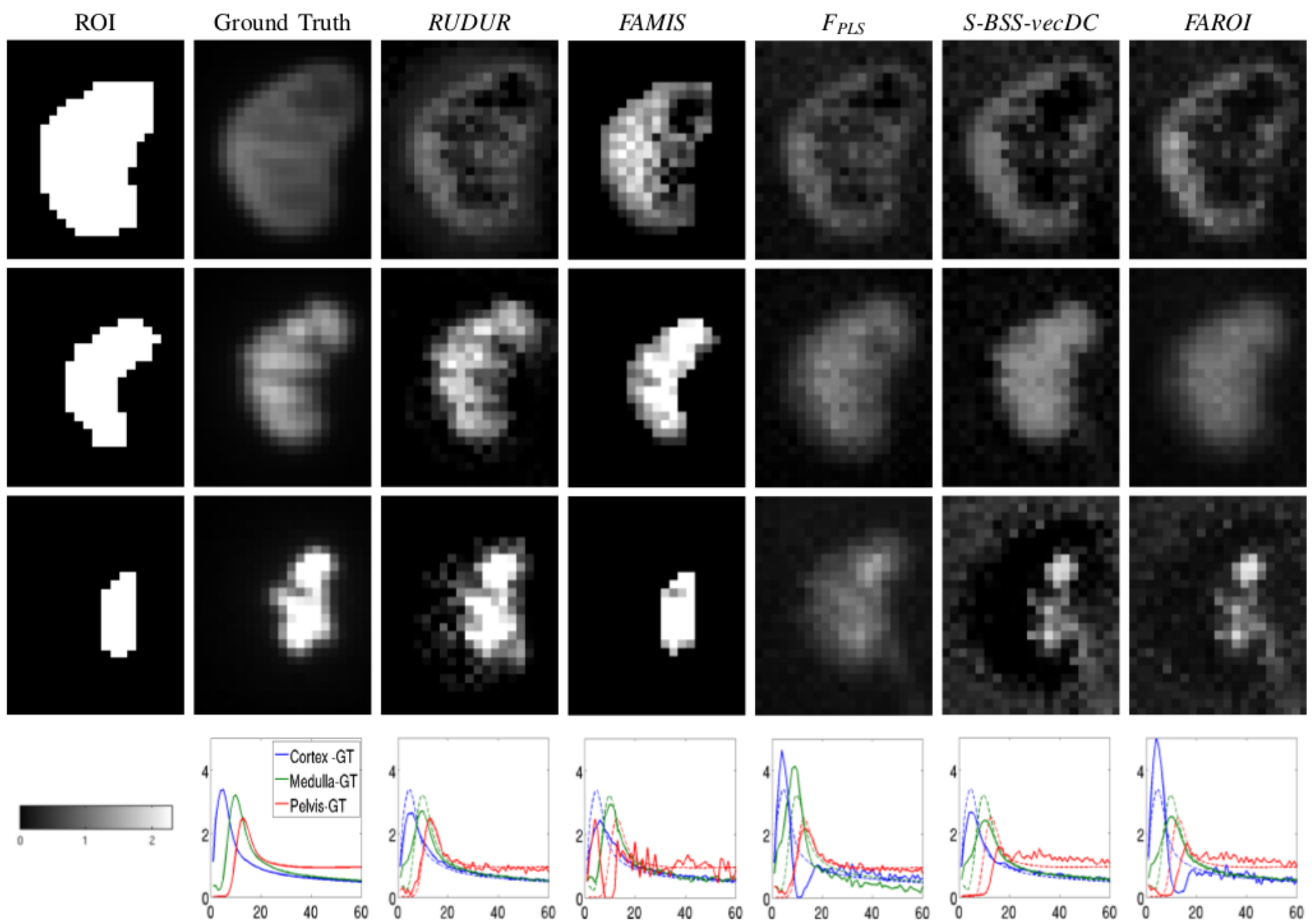

Fig. 3. Unmixing results on the highly realistic dataset (left kidney). The first three rows contain the ground truth (second column) and the estimation of source images for each algorithm (columns 3 to 7). Rows 1, 2 and 3 correspond to cortex, medulla and pelvis respectively. ROIs used for RUDUR and FAMIS are depicted in the first column. The colorbar used for the source images display is presented at the bottom left. The last row contains the ground truth (second column) and the estimation of TACs $(F)$ for each algorithm (columns 3 to 7). For columns 3 to 7, estimations are depicted in solid lines and the ground truth in dashed lines, with time on the $\mathrm{x}$-axis and activity on the $\mathrm{y}$-axis.

TABLE III

ESTIMATION ERRORS ON FACTORS $(F)$ - REALISTIC RENOGRAPHY

\begin{tabular}{|c|c|c|c|c|c|c|c|}
\hline \multirow[b]{2}{*}{ Measure } & \multirow[b]{2}{*}{ Algorithm } & \multicolumn{3}{|c|}{ Left Kidney } & \multicolumn{3}{|c|}{ Right Kidney } \\
\hline & & F1 & $\mathrm{F} 2$ & F3 & F1 & F2 & F3 \\
\hline \multirow{5}{*}{ NMSE } & RUDUR & $0.0327 \pm 0.0076$ & $0.0384 \pm 0.0088$ & $0.0302 \pm 0.0232$ & $0.0655 \pm 0.0217$ & $0.0419 \pm 0.0106$ & $0.0383 \pm 0.0155$ \\
\hline & FAMIS & $0.0559 \pm 0.0055$ & $0.0138 \pm 0.0051$ & $0.3996 \pm 0.1607$ & $0.0776 \pm 0.0083$ & $0.0514 \pm 0.0929$ & $0.1989 \pm 0.0372$ \\
\hline & $F_{P L S}$ & $0.1196 \pm 0.0417$ & $0.1481 \pm 0.1043$ & $0.0427 \pm 0.0278$ & $0.1093 \pm 0.0483$ & $0.0282 \pm 0.0093$ & $0.0610 \pm 0.0255$ \\
\hline & $S-B S S$ & $0.0374 \pm 0.0196$ & $0.1120 \pm 0.1174$ & $0.1755 \pm 0.0679$ & $0.0989 \pm 0.0858$ & $0.2047 \pm 0.3334$ & $0.1547 \pm 0.0678$ \\
\hline & FAROI & $0.2949 \pm 0.2354$ & $0.0546 \pm 0.0133$ & $0.2077 \pm 0.0637$ & $0.2314 \pm 0.1607$ & $0.0593 \pm 0.0222$ & $0.2458 \pm 0.0733$ \\
\hline \multirow{5}{*}{ NMAE } & RUDUR & $0.1303 \pm 0.0167$ & $0.1327 \pm 0.0137$ & $0.1356 \pm 0.0474$ & $0.1967 \pm 0.0374$ & $0.1411 \pm 0.0181$ & $0.1610 \pm 0.0377$ \\
\hline & FAMIS & $0.1699 \pm 0.0110$ & $0.0962 \pm 0.2206$ & $0.4415 \pm 0.0964$ & $0.2096 \pm 0.0162$ & $0.1593 \pm 0.1435$ & $0.3264 \pm 0.0360$ \\
\hline & $F_{P L S}$ & $0.2706 \pm 0.0484$ & $0.3169 \pm 0.1333$ & $0.1461 \pm 0.0394$ & $0.2645 \pm 0.0502$ & $0.1101 \pm 0.0181$ & $0.1774 \pm 0.0430$ \\
\hline & $S-B S S$ & $0.1672 \pm 0.0656$ & $0.2110 \pm 0.1334$ & $0.3014 \pm 0.0795$ & $0.2735 \pm 0.1334$ & $0.2812 \pm 0.2905$ & $0.2882 \pm 0.0860$ \\
\hline & FAROI & $0.3780 \pm 0.1382$ & $0.1486 \pm 0.0238$ & $0.3307 \pm 0.0535$ & $0.3857 \pm 0.1277$ & $0.1548 \pm 0.0398$ & $0.3764 \pm 0.0579$ \\
\hline
\end{tabular}

Results are in the form mean $\pm s t d$, where mean and std are the average and the standard deviation of the current measure (NMSE or NMAE) over a set of 6 sequences for each kidney (left and right). Each sequence has a different relative renal uptake and renal clearance rate. F1, F2 and F3 correspond to the TACs of the cortex, medulla and pelvis respectively. 
TABLE IV

ESTIMATION ERRORS ON SOURCE IMAGES $(A)$ - REALISTIC RENOGRAPHY

\begin{tabular}{|c|c|c|c|c|c|c|c|}
\hline & & & Left Kidney & & & Right Kidney & \\
\hline Measure & Algorithm & A1 & A2 & A3 & A1 & A2 & A3 \\
\hline \multirow{5}{*}{ NMSE } & $\bar{R}$ RUDUR & $0.2011 \pm 0.0462$ & $0.1283 \pm 0.0400$ & $0.0990 \pm 0.0405$ & $0.3072 \pm 0.1001$ & $0.1142 \pm 0.0201$ & $0.2234 \pm 0.1125$ \\
\hline & FAMIS & $0.7369 \pm 0.2930$ & $1.0194 \pm 0.1057$ & $1.4869 \pm 0.0911$ & $0.3306 \pm 0.0447$ & $0.8415 \pm 0.1055$ & $2.5143 \pm 0.4794$ \\
\hline & $F_{P L S}$ & $0.1945 \pm 0.0816$ & $0.1013 \pm 0.0336$ & $0.4274 \pm 0.0597$ & $0.2207 \pm 0.0865$ & $0.0913 \pm 0.0310$ & $0.6277 \pm 0.1394$ \\
\hline & $S-B S S$ & $0.4504 \pm 0.1797$ & $0.2553 \pm 0.3820$ & $0.5113 \pm 0.0944$ & $0.5177 \pm 0.2558$ & $0.3189 \pm 0.4990$ & $0.6807 \pm 0.1322$ \\
\hline & FAROI & $0.3215 \pm 0.0948$ & $0.1424 \pm 0.0674$ & $0.5499 \pm 0.1993$ & $0.3365 \pm 0.1554$ & $0.1788 \pm 0.0915$ & $0.7974 \pm 0.2013$ \\
\hline \multirow{5}{*}{ NMAE } & RUDUR & $0.4792 \pm 0.2011$ & $0.3691 \pm 0.1283$ & $0.4291 \pm 0.0990$ & $0.6242 \pm 0.3072$ & $0.3681 \pm 0.1142$ & $0.5766 \pm 0.2234$ \\
\hline & FAMIS & $0.7550 \pm 0.1055$ & $0.8686 \pm 0.0203$ & $1.0912 \pm 0.0079$ & $0.5190 \pm 0.0314$ & $0.7995 \pm 0.0124$ & $1.1967 \pm 0.0182$ \\
\hline & $F_{P L S}$ & $0.4723 \pm 0.1171$ & $0.3711 \pm 0.0686$ & $0.8898 \pm 0.0836$ & $0.5215 \pm 0.1133$ & $0.3568 \pm 0.0746$ & $1.1292 \pm 0.1368$ \\
\hline & $S-B S S$ & $0.7066 \pm 0.1656$ & $0.5396 \pm 0.4186$ & $1.1088 \pm 0.1475$ & $0.7721 \pm 0.2286$ & $0.6040 \pm 0.5058$ & $1.2398 \pm 0.1632$ \\
\hline & FAROI & $0.5985 \pm 0.0908$ & $0.4594 \pm 0.1196$ & $1.0923 \pm 0.1847$ & $0.6132 \pm 0.1433$ & $0.5171 \pm 0.1416$ & $1.2755 \pm 0.1567$ \\
\hline
\end{tabular}

Results are in the form mean $\pm s t d$ where mean and std are the average and the standard deviation of the current measure (NMSE or NMAE) over a set of 6 sequences for each kidney (left and right). Each sequence has a different relative renal uptake and renal clearance rate. A1, A2 and A3 correspond to the source images of the cortex, medulla and pelvis respectively. $S$-BSS corresponds to $S$-BSS-vecDC method.

to similar results to RUDUR when $\sigma=0.5$. This demonstrates the importance of ROIs knowledge. However FAMIS exhibits poor performance when $\sigma=1$. This can be incurred to the way ROIs knowledge is integrated as hard constraints in the FAMIS algorithm. Furthermore, source images produced by FAMIS remain mixed, which leads to higher estimation errors on source images.

For these results, we set $\alpha=1$ and $\beta=10$ for $R U D U R$. Setting $\alpha$ in [0.5 100] and $\beta$ in [1 200] does not significantly change the results. RUDUR still outperforms the other algorithms and results were even better when choosing $\alpha>1$. However, it should be mentionned that this dataset is not realistic, especially since source images are binarized.

\section{B. Highly realistic dynamic ${ }^{99 m}$ Tc-MAG3 renography}

Unlike first, the second dataset is highly realistic and contains Monte Carlo simulated data of dynamic renal study [28]. Both noisy and noiseless datasets can be retrieved from [29]. Noisy data includes effects of photon attenuation, scattering, limited spatial resolution and noise. The simulation was done using a whole body pharmacokinetic model with the XCAT phantom [30]. The aim of this experiment was to extract three factors from the kidney area (cortex, medulla and pelvis). This unmixing problem is very challenging because of both the noise and the presence of other structures in the kidney background. Furthermore, targeted factors are highly mixed. This highly realistic dataset is therefore a relevant case to compare algorithms performance.

The authors of the dataset provided us 6 noisy sequences along with the corresponding ground truth. Each sequence contains 60 images of size $128 \times 128$ pixels, and has a specific relative renal uptake and renal transit time. From these 6 sequences, 12 sequences of kidney (left and right) were extracted using a rectangular windows. Each extracted sequence was analysed with the same algorithms as in the toy example. As suggested in guidelines from [31], ROIs were drawn on the summed image. The values of constants $\alpha$ and $\beta$ were selected as in the toy example ( $\alpha=1$ and $\beta=10)$. Setting $\alpha$ in [0.2 2] and $\beta$ in [2 20] does not significantly change the results. Algorithms were compared using the ground truth provided by the authors of the dataset. A summary of results
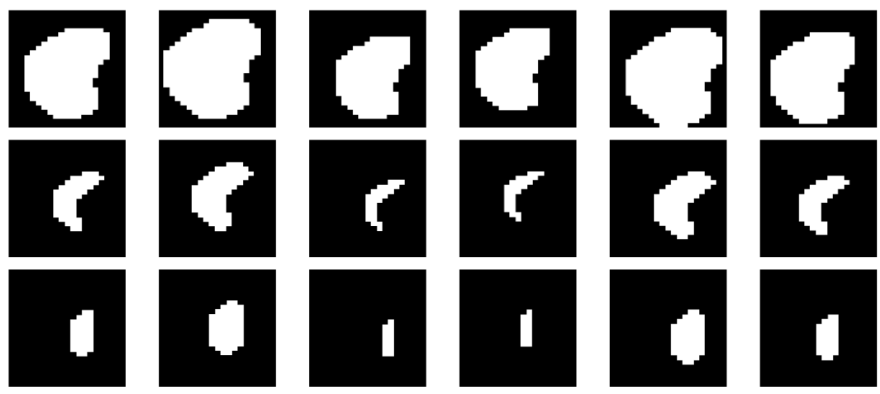

Fig. 4. Example of ROIs selected for robustness evaluation. Cortex ROIs are given in the first row, medulla ROIs in the second row, and pelvis ROIs in the third row. For each structure, the original ROI are eroded, dilated and both vertically and horizontally shifted. These modifications created 27 different ROIs combinations. Only 6 are presented on this figure.

obtained is presented in Tables III and IV, and visual results are depicted in Figure 3. The unmixing is greatly improved by RUDUR. It is the only method giving consistent estimation for all factors and for all simulations, as demonstrated by the low standard deviation of the results. In this dataset, source images are slightly fuzzy, mimicking the point spread function of a camera. The hard constraint used by FAMIS is not optimal in this more realistic context, because it does not allow source images to have low values outside of ROIs. The soft constraints set in $R U D U R$ are more permissive and more tolerant, which leads to a better unmixing for all sources. Thanks to the use of Tikhonov regularization, TACs provided by RUDUR are smooth unlike those provided by FAMIS. This regularization is very beneficial for datasets with high noise levels.

In order to evaluate the robustness to ROI selection, the previously used ROIs were modified by erosion, dilatation, and vertical and horizontal shifts. These modifications lead to 27 different ROIs $\left(3^{3}\right)$ for each structure (the cortex, the medulla and the pelvis) as depicted in Figure 4. RUDUR was applied on one kidney sequence with these 27 combinations of 3 ROIs. A summary of the results obtained is presented in Table V. As shown by the low standard deviation of estimation errors, modifications of the ROIs do not change significantly the results. This demonstrates robustness of the method to ROIs selection. 
TABLE V

ESTIMATION ERRORS OF RUDUR ON FACTORS $(F)$ AND SOURCE IMAGES $(A)$ ON ONE LEFT KIDNEY SEQUENCE OF REALISTIC RENOGRAPHY (27 DIFFERENT ROIS COMBINATIONS)

\begin{tabular}{|c|c|c|c|}
\hline Measure & $\mathrm{F} 1$ & $\mathrm{~F} 2$ & $\mathrm{~F} 3$ \\
\hline NMSE & $0.0426 \pm 0.0025$ & $0.0442 \pm 0.0097$ & $0.0187 \pm 0.0044$ \\
\hline NMAE & $0.1543 \pm 0.0037$ & $0.1509 \pm 0.0181$ & $0.1143 \pm 0.0120$ \\
\hline \hline Measure & $\mathrm{A} 1$ & $\mathrm{~A} 2$ & $\mathrm{~A} 3$ \\
\hline NMSE & $0.1880 \pm 0.0307$ & $0.1476 \pm 0.0621$ & $0.1109 \pm 0.0249$ \\
\hline NMAE & $0.4746 \pm 0.0503$ & $0.3990 \pm 0.0683$ & $0.4775 \pm 0.0577$ \\
\hline
\end{tabular}
Results are in the form mean \pm std where mean and std are the average
set of 27 different ROIs combinations. The low standard deviation
demonstrates the robustness of the proposed method to ROIs selection.

TABLE VI

ESTIMATION ERRORS ON 38 CORTICAL PARENCHYMA TACS (CLINICAL DATA)

\begin{tabular}{|c|c|c|}
\hline Algorithm & NMSE & NMAE \\
\hline RUDUR & $0.0111 \pm 0.0164$ & $0.0756 \pm 0.0368$ \\
\hline FAMIS & $0.0207 \pm 0.0369$ & $0.1004 \pm 0.0745$ \\
\hline$F_{P L S}$ & $0.0522 \pm 0.0679$ & $0.1523 \pm 0.1103$ \\
\hline$S-B S S$-vecDC & $0.0128 \pm 0.0085$ & $0.0875 \pm 0.0264$ \\
\hline FAROI & $0.0710 \pm 0.0818$ & $0.1804 \pm 0.1088$ \\
\hline
\end{tabular}

Results are in the form mean \pm std where mean and $s t d$ are the average and the standard deviation of the current measure (NMSE or NMAE) over a set of 38 cortical parenchyma TACs. Methods were compared with the currently recommended procedure guidelines from [31].

\section{Clinical dynamic ${ }^{99 m}$ Tc-MAG3 renography}

Finally, the methods were compared on a clinical dataset of dynamic renal studies, available at [29]. As in [19], 19 studies were selected, with clear visibility of the dynamic structure as criterion of choice. For each of the 38 kidneys, cortical parenchyma TAC were extracted with the recommended procedure guidelines from [31] and are used as ground truth. Clear visibility of dynamic structure is critical to have a consistent ground truth. Estimation errors were computed on the cortical parenchyma TACs only, since medulla and pelvis TACs cannot be reliably extracted without the use of a separation method. The same protocol with the same parameters as previous subsection was applied, and estimations errors were measured. Results are presented in Table VI. For a fair comparison, each TAC was previously normalized to have its sum equal to one before computing estimation errors.

For the clinical data, RUDUR and $S$-BSS-vecDC gave the best cortical parenchyma TACs estimation. An expert physician qualitatively assessed the relevance of the TACs and source images obtained. However, these results must be carefully interpreted. Indeed the parenchyma TACs used as ground truth are computed by averaging the signal of a ROI containing mainly but not only parenchyma, so they may not be perfectly reliable.

\section{CONCLUSION}

Structure separation in nuclear medicine is an underdetermined and challenging problem. The cost of an efficient unmixing is to use strong prior knowledge. In order to improve the separation, we proposed to use ROIs knowledge, which is an acceptable prior knowledge in nuclear medicine. A new unmixing method integrating ROIs knowledge as soft constraints was proposed. ROIs ensure a physiologically meaningful solution to be found, and greatly improve the unmixing. Through the use of a distance matrix, ROIs are robustly integrated in the unmixing procedure. The separation model is relaxed outside of the ROIs. Source images are constrained to be consistent with ROIs and the method promotes solutions with smooth factors. A significant improvement of the separation is observed for a toy example and for highly realistic and clinical renographies. Obviously, this is not the only way to introduce ROIs knowledge. For example, the combination of ROIs with a physiological model in a Bayesian framework as in $S B S S$-vec-DC method can be considered.

In FAMIS [21], it is possible to integrate additional prior knowledge on the physical model, for example fixed coefficients in source images or TACs. This can be done in RUDUR by forcing the corresponding partial derivatives to zero. This was not considered in this paper, as we focused on the use of ROIs knowledge.

This paper demonstrates the potential of ROIs knowledge for the source separation problem in nuclear medicine. A generic and efficient method was developed based on ROIs knowledge. In this method, there is no predetermined shape for the ROIs, making it flexible and usable in a wide variety of situations. Also the method can be efficient in challenging and noisy conditions. The proposed method can be applied in scintigraphy, but also in similar source separation problems, where ROIs are easily available and located in a specific area. We are currently working on a 3D-dataset (SPECT), with 6-DIG tracer, able to compute insulin-resistance index [32]. In order to compute this index, it is necessary to unmix myocardium and blood activities in a challenging and very noisy context, where traditional methods failed.

Since the ROIs have to be chosen manually, the proposed method is only semi-automatic. However, thanks to the distance to ROIs matrix, RUDUR is robust to ROIs selection. An automatic choice of coarse ROIs can thus be considered to avoid the manual selection.

\section{APPENDIX A}

PARTIAL DERIVATIVES

$$
\begin{aligned}
& \frac{\partial f_{\mathrm{WLS}}}{\partial A}(A, F)=-2 W^{2}(Y-A F) F^{T} \\
& \frac{\partial f_{\mathrm{WLS}}}{\partial F}(A, F)=-2 A^{T} W^{2}(Y-A F)
\end{aligned}
$$

$$
\begin{gathered}
\frac{\partial f_{\mathrm{ROI}}}{\partial A_{i, k}}(A)=\frac{D_{i, k}^{4} A_{i, k}}{\sqrt{D_{i, k}^{4} A_{i, k}^{2}+\mu^{2}}} \\
f_{\text {Tik }}^{\prime}(F)=2 F\left(\Gamma^{T} \Gamma\right)
\end{gathered}
$$




\section{APPENDIX B}

\section{LIPSCHITZ CONSTANT}

For a given $F$ (resp. A), we note $f_{O b j_{F}}$ (resp. $f_{O b j_{A}}$ ) the partial function $A \mapsto f_{\text {RUDUR }}(A, F)$ (resp. $F \mapsto$ $f_{R U D U R}(A, F)$. Their derivatives are Lipshitz continuous functions, with

$$
\frac{\left\|f_{O b j_{A}}^{\prime}(F)-f_{O b j_{A}}^{\prime}\left(F^{\prime}\right)\right\|_{F}}{\left\|F-F^{\prime}\right\|_{F}} \leq 2\left(\left\|A^{T} W^{2} A\right\|_{F}+\beta\left\|\Gamma^{T} \Gamma\right\|_{F}\right)
$$

If we approximate the smooth $\ell 1$-norm in $f_{R O I}$ by the classic $\ell$ 1-norm $(\mu=0)$, then $f_{\mathrm{ROI}}^{\prime}(A)=D \circ D$. We then obtain a Lipschitz constant for each pixel $i$ such that

$$
\frac{\left\|\frac{\partial f_{O b j_{F}}}{\partial A_{i}}\left(A_{i}\right)-\frac{\partial f_{O b j_{F}}}{\partial A_{i^{\prime}}}\left(A_{i^{\prime}}\right)\right\|_{F}}{\left\|A_{i}-A_{i^{\prime}}\right\|_{F}} \leq 2 W_{i, i}\left\|F F^{T}\right\|_{F}
$$

\section{ACKNOWLEDGMENT}

The authors would like to thank G.Brolin of Lund University for providing us the ground truth of his dataset.

\section{REFERENCES}

[1] R. Di Paola, J. Bazin, F. Aubry, A. Aurengo, F. Cavailloles, J. Herry, and E. Kahn, "Handling of dynamic sequences in nuclear medicine," IEEE Transactions on Nuclear Science, vol. 29, no. 4, pp. 1310-1321, 1982.

[2] G. T. Gullberg, B. W. Reutter, A. Sitek, J. S. Maltz, and T. F. Budinger, "Dynamic single photon emission computed tomographybasic principles and cardiac applications," Physics in medicine and biology, vol. 55, no. 20, p. R111, 2010.

[3] M. Abdalah, R. Boutchko, D. Mitra, and G. T. Gullberg, "Reconstruction of 4-d dynamic spect images from inconsistent projections using a spline initialized fads algorithm (sifads)," IEEE transactions on medical imaging, vol. 34, no. 1, pp. 216-228, 2015.

[4] H.-M. Wu, C. K. Hoh, Y. Choi, H. R. Schelbert, R. A. Hawkins, M. E. Phelps, and S.-C. Huang, "Factor analysis for extraction of blood timeactivity curves in dynamic fdg-pet studies." Journal of nuclear medicine: official publication, Society of Nuclear Medicine, vol. 36, no. 9, pp. 1714-1722, 1995.

[5] J. Y. Ahn, D. S. Lee, J. S. Lee, S.-K. Kim, G. J. Cheon, J. S. Yeo, S.-A. Shin, J.-K. Chung, and M. C. Lee, "Quantification of regional myocardial blood flow using dynamic h215o pet and factor analysis," Journal of Nuclear Medicine, vol. 42, no. 5, pp. 782-787, 2001.

[6] L. Chen, P. L. Choyke, T.-H. Chan, C.-Y. Chi, G. Wang, and Y. Wang, "Tissue-specific compartmental analysis for dynamic contrast-enhanced $\mathrm{mr}$ imaging of complex tumors," IEEE transactions on medical imaging, vol. 30, no. 12, pp. 2044-2058, 2011.

[7] G. J. Lueck, T. Kim, P. N. Burns, and A. L. Martel, "Hepatic perfusion imaging using factor analysis of contrast enhanced ultrasound," IEEE transactions on medical imaging, vol. 27, no. 10, pp. 1449-1457, 2008.

[8] J. Zhang, M. Ding, F. Meng, and X. Zhang, "Quantitative evaluation of two-factor analysis applied to hepatic perfusion study using contrastenhanced ultrasound," IEEE Transactions on Biomedical Engineering, vol. 60, no. 2, pp. 259-267, 2013.

[9] D. Barber, "The use of principal components in the quantitative analysis of gamma camera dynamic studies," Physics in medicine and biology, vol. 25 , no. 2, p. $283,1980$.

[10] P. Paatero and U. Tapper, "Positive matrix factorization: A non-negative factor model with optimal utilization of error estimates of data values," Environmetrics, vol. 5, no. 2, pp. 111-126, 1994.

[11] D. D. Lee and H. S. Seung, "Algorithms for non-negative matrix factorization," in Advances in neural information processing systems, 2001, pp. 556-562.

[12] A. Houston, "The effect of apex-finding errors on factor images obtained from factor analysis and oblique transformation (nuclear medicine)," Physics in medicine and biology, vol. 29, no. 9, p. 1109, 1984.
[13] M. Samal, M. Karny, H. Surova, P. Penicka, E. Marikova, and Z. Dienstbier, "On the existence of an unambiguous solution in factor analysis of dynamic studies," Physics in medicine and biology, vol. 34, no. 2, p. $223,1989$.

[14] B. W. Reutter, G. T. Gullberg, and R. H. Huesman, "Direct leastsquares estimation of spatiotemporal distributions from dynamic spect projections using a spatial segmentation and temporal b-splines," IEEE transactions on medical imaging, vol. 19, no. 5, pp. 434-450, 2000.

[15] T. E. Nichols, J. Qi, and R. M. Leahy, "Continuous time dynamic pet imaging using list mode data," in Biennial International Conference on Information Processing in Medical Imaging. Springer, 1999, pp. 98111.

[16] F. Frouin, A. De Cesare, Y. Bouchareb, A. Todd-Pokropek, and A. Herment, "Spatial regularization applied to factor analysis of medical image sequences (famis)," Physics in medicine and biology, vol. 44, no. 9, p. 2289, 1999.

[17] A. Sitek, G. T. Gullberg, and R. H. Huesman, "Correction for ambiguous solutions in factor analysis using a penalized least squares objective," IEEE transactions on medical imaging, vol. 21, no. 3, pp. 216-225, 2002.

[18] V. Šmídl and O. Tichỳ, "Sparsity in bayesian blind source separation and deconvolution," in Joint European Conference on Machine Learning and Knowledge Discovery in Databases. Springer, 2013, pp. 548-563.

[19] O. Tichỳ and V. Šmídl, "Bayesian blind separation and deconvolution of dynamic image sequences using sparsity priors," IEEE transactions on medical imaging, vol. 34, no. 1, pp. 258-266, 2015.

[20] K. Nijran and D. Barber, "The importance of constraints in factor analysis of dynamic studies," in Information processing in medical imaging. Springer, 1988, pp. 521-529.

[21] H. Benali, I. Buvat, F. Frouin, J. Bazin, and R. Di Paola, "Foundations of factor analysis of medical image sequences: a unified approach and some practical implications," Image and Vision Computing, vol. 12, no. 6, pp. 375-385, 1994.

[22] V. Šmídl and O. Tichỳ, "Automatic regions of interest in factor analysis for dynamic medical imaging," in 2012 9th IEEE International Symposium on Biomedical Imaging (ISBI). IEEE, 2012, pp. 158-161.

[23] M. Filippi, M. Desvignes, E. Moisan, C. Ghezzi, P. Perret, and D. Fagret, "Factor analysis of dynamic sequence with spatial prior for $2 \mathrm{~d}$ cardiac spect sequences analysis," in International Conference on Advanced Concepts for Intelligent Vision Systems. Springer, 2016, pp. 228-237.

[24] _ , "A priori spatiaux et analyse factorielle de séquences scintigraphiques," in Proceedings du GRETSI 15, 2015, pp. 1-4.

[25] M. W. Berry, M. Browne, A. N. Langville, V. P. Pauca, and R. J. Plemmons, "Algorithms and applications for approximate nonnegative matrix factorization," Computational statistics \& data analysis, vol. 52, no. 1, pp. 155-173, 2007.

[26] E. Polak and G. Ribiere, "Note sur la convergence de méthodes de directions conjuguées," Revue française d'informatique et de recherche opérationnelle, série rouge, vol. 3, no. 1, pp. 35-43, 1969.

[27] V. Šmídl and A. Quinn, The variational Bayes method in signal processing. Springer Science \& Business Media, 2006.

[28] G. Brolin, K. S. Gleisner, and M. Ljungberg, "Dynamic 99mtc-mag3 renography: images for quality control obtained by combining pharmacokinetic modelling, an anthropomorphic computer phantom and monte carlo simulated scintillation camera imaging," Physics in medicine and biology, vol. 58, no. 10, p. 3145, 2013.

[29] (2014) Database of dynamic renal scintigraphy. [Online]. Available: http://www.dynamicrenalstudy.org

[30] W. Segars, G. Sturgeon, S. Mendonca, J. Grimes, and B. M. Tsui, " $4 \mathrm{~d}$ xcat phantom for multimodality imaging research," Medical physics, vol. 37, no. 9, pp. 4902-4915, 2010.

[31] I. Gordon, A. Piepsz, and R. Sixt, "Guidelines for standard and diuretic renogram in children," European journal of nuclear medicine and molecular imaging, vol. 38, no. 6, pp. 1175-1188, 2011.

[32] P. Perret, M. Henri, L. Slimani, D. Fagret, and C. Ghezzi, "Nuclear imaging of glucose transport/metabolism-an interesting tool to screen insulin resistance, refine diagnosis of type 2 diabetes, understand disease mechanisms, and/or evaluate new therapies," in Medical Complications of Type 2 Diabetes. InTech, 2011. 